\title{
Bone Marrow Foot Oedema in Adolescents: The Role of Vitamin D
}

\author{
Sara Pieropan' ', Franco Antoniazzi', Elisa Tadiotto', Federico Caldonazzi ', Maddalena Maschio', Giulia Aiello', \\ Giulia Melotti' ${ }^{1}$ Paolo Cavarzere' ${ }^{1}$, Giorgio Piacentini' \\ 'Pediatric Clinic, Department of Life and Reproduction Sciences, Azienda Ospedaliera Universitaria Integrata, Verona; \\ ${ }^{2}$ Pediatric Clinic, Santa Chiara Hospital, Trento, Italy
}

Corresponding author

Elisa Tadiotto

Pediatric Clinic, Department of Life and

Reproduction Sciences, Azienda Ospedaliera

Universitaria Integrata,

Piazzale Aristide Stefani, 1, Verona 37126,

Italy

Tel: +39-45-812-7857

Fax: +39-45-812-7830

E-mail: elisatadiotto@yahoo.it

Received: July 25, 2019

Revised: October 31, 2019

Accepted: November 1, 2019
Background: Bone marrow oedema (BMO) in children/adolescents is a rare clinical condition without an etiologic cause. It is associated with typical increased signal intensity on T2-weighted magnetic resonance images (MRI) and an increase in bone turnover in which vitamin $D$ plays a pivotal role. No treatment guidelines for these young patients are to date available. Methods: We performed a retrospective study in a pediatric setting of 13 patients with diagnosis of primary BMO of the foot on the basis of clinical and radiological findings. Data collection included sex, age, patient history, symptoms at presentation, clinical examination, laboratory bone turnover markers, vitamin D levels, $\mathrm{MRI}$, treatment, and outcome. Results: Vitamin D deficiency or insufficiency was found in $76.9 \%$ of cases. All patients were treated with adequate vitamin D daily intake, a short course of analgesic therapy, physical therapy, avoiding detrimental feet and ankle immobilization. All fully recovered in 3-month lag period. Conclusions: Our data highlight that environmental factors, such as underestimated articular or bone microtraumatisms, as well as joint hyper mobility, in a bone turnover milieu of vitamin D deficiency could be the cause of this clinical conditions. Adequate vitamin D supplementation, associated with physical and analgesic therapy, is crucial in the management of BMO.

Key Words: Bone marrow oedema $\cdot$ Child $\cdot$ Magnetic resonance imaging $\cdot$ Vitamin $D$

\section{INTRODUCTION}

Primary bone marrow oedema $(\mathrm{BMO})$ is a rare clinical condition characterized by joint and bone extremity pain, out of proportion to the clinical findings, exacerbated by weight bearing, in the absence of a known etiologic cause.[1-5] These clinical symptoms are associated with typical increased signal intensity on T2-weighted magnetic resonance images (MRI), without evidence of focal specific signs of osteonecrosis, fracture, neoplasm, or infections.[1-4] This condition has been first described by Wilson et al.[6], who found ill-defined bone marrow hyperintensities on T2 weighted MRI in 3 patients with knee pain of no apparent cause. Since then, various terms have been used to describe this entity; in fact transient osteoporosis, transient BMO syndrome, and migratory osteoporosis have all been used interchangeably.[1,2] BMO could be a feature of other conditions (secondary BMO); among them trauma, inflammatory conditions (e.g., arthritis, enthesitis), infec-
Copyright (c) 2019 The Korean Society for Bone and Mineral Research

This is an Open Access article distributed under the terms of the Creative Commons Attribution Non-Commercial License (http://creativecommons.org/licenses/by-nc/4.0/) which permits unrestricted non-commercial use, distribution, and reproduction in any medium, provided the origina work is properly cited.

\section{KSBMR}


tious diseases (e.g., septic arthritis, osteomyelitis), ischaemic events (e.g., sickle cell disease, polycythaemia), neoplasm, degenerative disorders, neurologic disorders (e.g., Charcot arthropathy), metabolic/endocrine disease, and iatrogenic causes (e.g., drugs such calcineurin inhibitor or steroids, after radiotherapy or surgery).[1-3,5] Therefore, the diagnosis of primary $\mathrm{BMO}$ is made after the exclusion of these pathologies.[1-4,7]

The multiple names and the fact that primary BMO is diagnosis by exclusion, reflect the uncertainty about its aetiology.[1-3,8] It mostly affects middle-aged men (range, 3060 years) and younger women (range, 20-40 years).[3] The most commonly affected sites are bone of the hip, knee, ankle and foot.[1,2] BMO is also rarely described in children/ adolescents, even if the incidence is unknown.[1,4,7] It has been suggested that mechanical, vascular, inflammatory or metabolic trauma may initiate a chain of events resulting in increased intraosseous pressure, irritation of sensory nerves within the bone marrow, periosteum and periarticular structures. These lead to bone damage and BMO.[1-3] Clinically it manifests with pain, sometimes irritable joint or mild subcutaneous oedema of ankle or foot; but trophic or vasomotor changes are absent.[3] Pain usually improves within 3 to 9 months without treatment, although the course could be longer, up to 24 months. [2,9]

Treatment has mostly been reported in adult case series (corticosteroids, bisphosphonates, vasodilators, physiotherapy, reduction of weight-bearing, core decompression), but randomized controlled trials are lacking and no treatment guidelines for younger patients are available.[1-4,10,11] Recently, it has become evident that BMO is accompanied by an increase in bone turnover, in which vitamin $D$ plays a pivotal role. Vitamin D deficiency and insufficiency negatively affect bone mineralization.[1,6,11-15] To date, limited information is available about vitamin $D$ status in patients with BMO. Sprinchorn et al.[16] and Horas et al.[1] reported an association between hypovitaminosis $D$ and $\mathrm{BMO}$ of the foot and ankle in small adult case series. No data are reported in cohorts of children and adolescents.

The purpose of this study is to investigate the incidence of hypovitaminosis $D$ in a young aged population with primary BMO of the foot and the benefit of a vitamin D supplementation therapy.

\section{METHODS}

A retrospective study has been performed in a paediatric setting of 13 patients with persistent foot pain and MRI showing a picture compatible with bone oedema of the foot, referred to our Rheumatologic Paediatric Clinic in the period of 2015 to 2018. They are all misdiagnosed in other institutions as affected by algodystrophy or complex regional pain syndrome.

This study included patients with age $\leq 18$ years, affected by primary BMO of the foot. The diagnosis of BMO was based on patient's medical history and clinical examination (sudden onset and persistent foot pain), and on the presence of ill-defined abnormal bone marrow hyperintensities on T2 weighted MRI. Exclusion criteria encompassed age $>18$ years, MRI demonstrating other concomitant diagnosis affecting the bone (e.g., neoplasia, fractures, infections), the presence of other pathologies causing secondary $\mathrm{BMO}$ and patients lost during the follow up.

Data collection included sex, age, medical and surgical history, recent or remote trauma history, symptoms at presentation, clinical examination, laboratory bone turnover markers (if available), vitamin D levels, MRI, treatment and the outcome. Prevalence of hypovitaminosis D in this case series was evaluated.

Concerning vitamin $D$ levels, to date there is no universally accepted classification of vitamin D deficiency and insufficiency according to the serum level of 25-hydroxy-vitamin D $(25[\mathrm{OH}]$ D). We used Endocrine Society Clinical Practice Guidelines defining vitamin $D$ deficiency as $25(\mathrm{OH}) \mathrm{D}$ level of $<20 \mathrm{ng} / \mathrm{mL}$ ( 50 $\mathrm{nmol} / \mathrm{L}$ ), vitamin $\mathrm{D}$ insufficiency as $25(\mathrm{OH}) \mathrm{D}$ level between 20 and $30 \mathrm{ng} / \mathrm{mL}$ (50-75 nmol/L) and vitamin D sufficiency as 25(OH)D level $\geq 30 \mathrm{ng} / \mathrm{mL}$ (75 nmol/L).[13] Serum 25(OH)D was measured by a immunochemiluminescent assay (LIAISON ${ }^{\circledR}$, Diasorin; DiaSorin Ltd, Schiphol Rijk, The Netherlands).

This is a retrospective study, the investigations are based on preexisting clinical practice so ethical permission and informed consent are unnecessary. The study is performed following the Declaration of Helsinki and under the terms of relevant local legislation.

\section{RESULTS}

\section{Clinical features of the 13 patients evaluated are reported in Table 1 \\ Eleven/13 are female (male to female ratio 1:5.5). The}




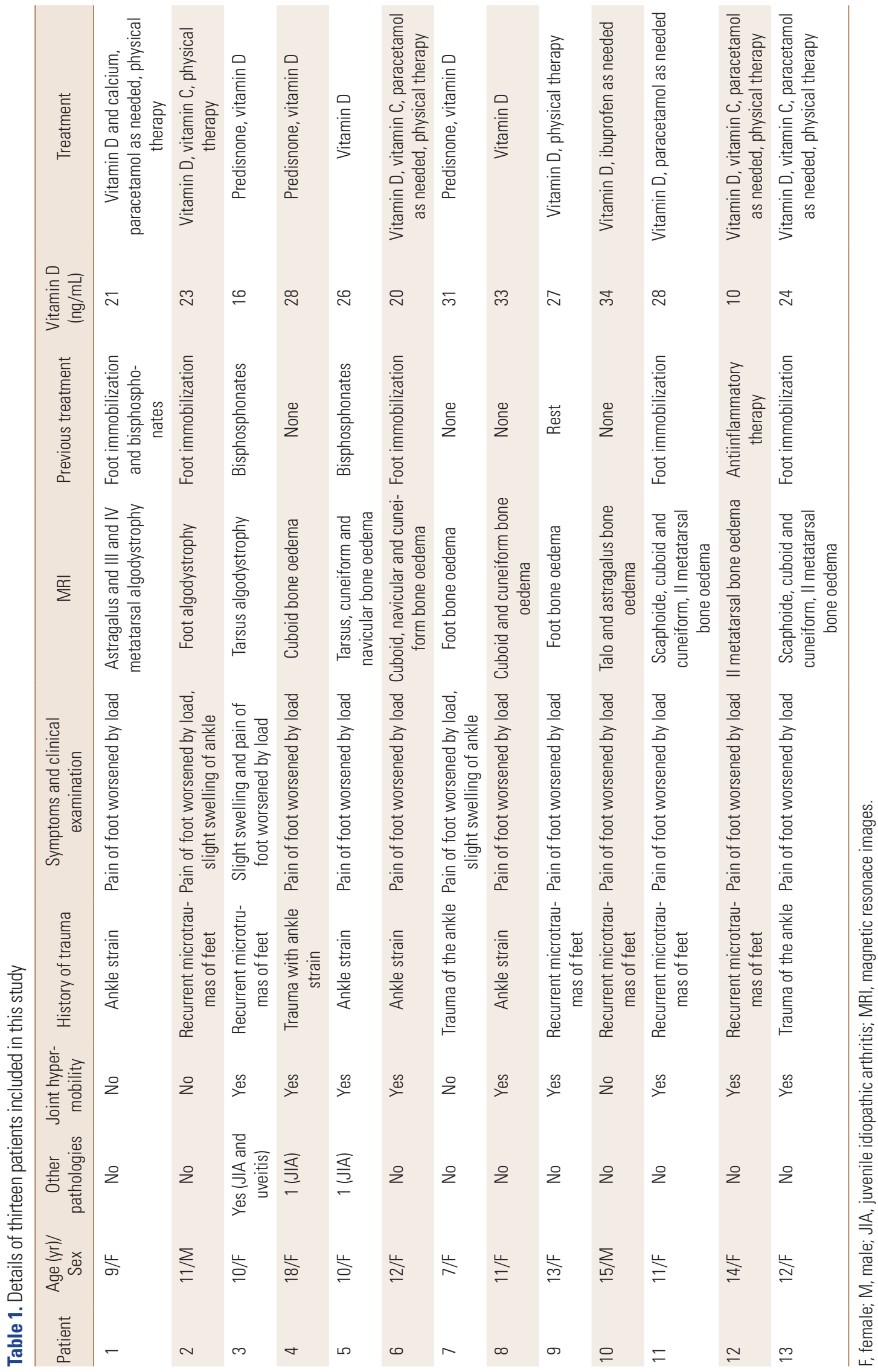


mean age is 11.8 years (range, 7-18 years). All patients live near to Verona, in the Veneto region of northern Italy (latitude $45.26^{\circ} \mathrm{N}$, longitude $19.59^{\circ} \mathrm{E}$ ). Eleven $/ 13$ patients are Italians, $1 / 13$ is Moroccan, $1 / 13$ of Moroccan ethnicity was born and raised in Italy.

Three/13 had a previous diagnosis of juvenile idiopathic arthritis with positivity of antinuclear antibodies, but the disease was in complete remission at our evaluation. 10/13 were previously healthy.

In all cases history of minor ankle strain or recurrent microtraumas of feet prior to symptom onset had been reported after accurate interview. In particular, $7 / 13$ patients reported an ankle strain or a major trauma; $6 / 13$ had history of probable microtraumatism mainly due to sport activity.

In all cases the symptom at onset was pain. Clinical examination confirmed pain of foot and ankle, worsened by joint load. Two patients presented slight swelling of ankle or foot. No one presented hyperemia, heat, trophic or vasomotor change of the feet. Joint hypermobility was observed in $69.2 \%$ of cases.

The $77 \%$ of cases presented multiple lesions at MRI, while $23 \%$ of patients presented a single lesion. Five/13 patients were treated with foot immobilization before our evaluation. 3 patients were previously treated with bisphosphonates, without improvement.

Vitamin D deficiency or insufficiency was found in 10/13 cases $(76.9 \%)$, deficiency in $3 / 13$ patients $(23.1 \%)$, insufficiency in $7 / 13$ patients (53.8\%). In 3/13 patients (23.1\%) vitamin D was sufficient but at lower limits (range, $31-34 \mathrm{ng} /$ $\mathrm{mL}$ ). Medium 25(OH)D level is $24.7 \mathrm{ng} / \mathrm{mL} \pm 2.1$ (range, 10$34 \mathrm{ng} / \mathrm{mL}$ ). Inflammatory indices (leukocytes, reactive $C$ protein, erythrocyte sedimentation rate) were normal in all cases. We have data on parathyroid hormone (PTH) status for 8 patients, it was high in 1 patient $(44 \mathrm{pg} / \mathrm{mL})$, normal in 7 patients, among them PTH was at the upper level of the normal range in 2 patients. Serum calcium levels, measured in 11/13 patients, were normal. Bone alkaline phosphatase had been measured in 6/13 patients, in all patients it was high (medium value of $136,5 \mu \mathrm{g} / \mathrm{L}$ ). Osteocalcin, measured in 3 patients, was high in 2 cases, normal in one patient. C-terminal telopeptide of type I collagen (CTX) had been measured in 3 patients and it was high in all patients (medium value of $2,3 \mathrm{ng} / \mathrm{mL}$ ).

All patients were treated with adequate vitamin $D$ daily intake (therapeutic dose and then maintenance dose, ac- cording to current guidelines). $[1,13,14]$ Pain relief was achieved with paracetamol, low dose ibuprofen, or a short course of oral prednisone. Rest from intense physical activity, physical exercises to promote the ability to move the foot and/ or the ankle, avoiding detrimental feet and ankle immobilization were recommended. All children (100\%) fully recovered in 3-month lag period.

\section{DISCUSSION}

Vitamin D deficiency or insufficiency is one of the most prevalent nutritional deficiency in the world and it has been estimated to affect more than 1 billion people globally.[1, $13,14]$ In Europe almost $80 \%$ of adolescents have been found to have a hypovitaminosis D.[13,17] The prevalence of hypovitaminosis $D$ in our case series is not so different from that of other studies.[18-22] Vitamin D and adequate calcium intake are crucial for bone health and for a balanced bone turnover. The $40 \%$ to $50 \%$ of skeletal mass is accumulated in childhood.[14] Vitamin D deficiency in childhood and adolescence is associated with suboptimal peak bone mass and lower bone mineral density, leading to insufficient bone mineralization and, as consequence, increased bone fragility and reduced self-repairing capacity of the bone tissue. $[8,11,15]$ This causes not only osteoporosis, rickets, osteomalacia, but also aggravation of many orthopaedic and bone disease patterns, among them BMO.[11,13-15,23] Adolescence is a critical period because pubertal growth leads to a phase of accelerated bone turnover, which could predispose to bone microdamage even under physiological load. [8] Environmental factors, such as underestimated articular or bone microtraumatisms, as well as joint hyper mobility, typical of paediatric age, in a bone turnover milieu of hypovitaminosis $\mathrm{D}$, could lead to the development of $\mathrm{BMO}$.

$\mathrm{BMO}$ in pediatric age is uncommon, but it is an important cause of disability. It is often misdiagnosed as complex regional pain syndrome or algodystrophic syndrome manifestation. $[7,24]$ To date there are few reports in literature about primary BMO in paediatric patients.[4,9,25-29] Like in adult, BMO lesions affect weight-bearing and thus load affected joints: more frequently foot and ankle, $[4,6,9]$ but also acetabulum,[26] distal tibia and fibula,[4,25] femoral head,[27,29] knee and hip.[28] In the majority of these reports serum levels of vitamin $D$ have not been analysed. Kröger et al.[4] reported normal vitamin D levels, with sli- 
ghtly augmented bone turnover markers. Kaspiris et al.[9] reported association with vitamin $D$ deficiency in a 12-yearold girl with primary BMO of the foot, like in our case. In these reports treatment is focused mostly on symptomatic relief, such as activity limitation, avoidance of weight bearing, protective boot application, analgesic drugs. In one case vasodilatator treatment has been used,[26] but the possible side effects (e.g., headache, nausea, flushing), made this choice unlikely.[9] Only in one case a successful treatment with vitamin $\mathrm{D}$ administration, in order to correct hypovitaminosis $D$, has been reported.[9]

Our data are in accordance with the findings in the adult case series reported by Sprinchorn et al.[16] and Horas et al.[1]. They described a series of 9 and 31 middle aged patients with primary BMO; vitamin D deficiency or insufficiency had been detected in $90 \%$ of cases. In our case series hypovitaminosis $D$ has been detected in $77 \%$ of cases, in the remaining $23 \%$ vitamin $D$ was sufficient but at lower limits. Although serum calcium and PTH levels were in normal range except for one case, bone alkaline phosphatase and CTX (when available) where raised, reflecting ah increased bone turnover.

Further studies are necessary to establish the precise role of vitamin D supplementation in the improvement of BMO in children as well as possible role of the association of medication (e.g., pharmacological therapy with vitamin D supplementation). Certainly our results highlight that adequate vitamin D supplementation, associated with physical and analgesic therapy, is crucial in the management of BMO. Paediatricians should be aware of the diagnosis and the management, in order to promptly begin an adequate treatment. All patients with primary BMO should be screened for bone metabolism disorders, especially vitamin $D$ levels. [8] Delay in diagnosis, unnecessary diagnostic testing and procedures with incorrect or delayed treatment, lead to worsening the discomfort of the patients.

Furthermore, we underline the importance of supplementing vitamin $D$ as recommended in the current guidelines, $[1,13,14]$ in order to avoid its deficiency and prevent invalidating clinical complaints.

\section{DECLARATIONS}

\section{Ethics approval and consent to participate}

This study was conducted in accordance with the princi- ples of the Declaration of Helsinki.

\section{Conflict of interest}

No potential conflict of interest relevant to this article was reported.

\section{ORCID}

Franco Antoniazzi https://orcid.org/0000-0003-1405-1792

Elisa Tadiotto https://orcid.org/0000-0003-0366-7348

Paolo Cavarzere https://orcid.org/0000-0002-2386-7307

Giorgio Piacentini https://orcid.org/0000-0003-1324-4373

\section{REFERENCES}

1. Horas K, Fraissler L, Maier G, et al. High prevalence of vitamin $D$ deficiency in patients with bone marrow edema syndrome of the foot and ankle. Foot Ankle Int 2017;38: 760-6.

2. Mirghasemi SA, Trepman E, Sadeghi MS, et al. Bone marrow edema syndrome in the foot and ankle. Foot Ankle Int 2016;37:1364-73.

3. Patel S. Primary bone marrow oedema syndromes. Rheumatology (Oxford) 2014;53:785-92.

4. Kröger L, Arikoski P, Komulainen J, et al. Transient bone marrow oedema in a child. Ann Rheum Dis 2004;63:1528-9.

5. Korompilias AV, Karantanas AH, Lykissas MG, et al. Bone marrow edema syndrome. Skeletal Radiol 2009;38:42536.

6. Wilson AJ, Murphy WA, Hardy DC, et al. Transient osteoporosis: transient bone marrow edema? Radiology 1988;167: 757-60.

7. Orr JD, Sabesan V, Major N, et al. Painful bone marrow edema syndrome of the foot and ankle. Foot Ankle Int 2010; 31:949-53.

8. Oehler N, Mussawy H, Schmidt T, et al. Identification of vitamin $\mathrm{D}$ and other bone metabolism parameters as risk factors for primary bone marrow oedema syndrome. BMC Musculoskelet Disord 2018;19:451.

9. Kaspiris A, Savvidou OD, Chrronopoulos E, et al. Juvenile transient bone marrow oedema of the foot associated with Vitamin D deficiency: A case study and an overview of pathogenesis and treatment. Foot (Edinb) 2019;38:50-3.

10. Radke S, Vispo-Seara J, Walther M, et al. Transient bone marrow oedema of the foot. Int Orthop 2001;25:263-7.

11. Berger $\mathrm{CE}$, Kroner $\mathrm{AH}$, Minai-Pour MB, et al. Biochemical 
markers of bone metabolism in bone marrow edema syndrome of the hip. Bone 2003;33:346-51.

12. Simon MJ, Barvencik F, Luttke M, et al. Intravenous bisphosphonates and vitamin $\mathrm{D}$ in the treatment of bone marrow oedema in professional athletes. Injury 2014;45:981-7.

13. Saggese G, Vierucci F, Boot AM, et al. Vitamin D in childhood and adolescence: an expert position statement. Eur J Pediatr 2015;174:565-76.

14. Randev S, Kumar P, Guglani V. Vitamin D supplementation in childhood - A review of guidelines. Indian J Pediatr 2018; 85:194-201.

15. Maier GS, Horas K, Seeger JB, et al. Is there an association between periprosthetic joint infection and low vitamin $D$ levels? Int Orthop 2014;38:1499-504.

16. Sprinchorn AE, O'Sullivan R, Beischer AD. Transient bone marrow edema of the foot and ankle and its association with reduced systemic bone mineral density. Foot Ankle Int 2011;32:S508-12.

17. González-Gross M, Valtuena J, Breidenassel C, et al. Vitamin D status among adolescents in Europe: the Healthy Lifestyle in Europe by Nutrition in Adolescence study. Br J Nutr 2012;107:755-64.

18. Franchi B, Piazza M, Sandri M, et al. 25-hydroxyvitamin D serum level in children of different ethnicity living in Italy. Eur J Pediatr 2015;174:749-57.

19. Saggese G, Vierucci F, Prodam F, et al. Vitamin D in pediatric age: consensus of the Italian Pediatric Society and the Italian Society of Preventive and Social Pediatrics, jointly with the Italian Federation of Pediatricians. Ital J Pediatr
2018;44:51.

20. Kumar J, Muntner P, Kaskel FJ, et al. Prevalence and associations of 25-hydroxyvitamin D deficiency in US children: NHANES 2001-2004. Pediatrics 2009;124:e362-70.

21. Mansbach JM, Ginde AA, Camargo CA Jr. Serum 25-hydroxyvitamin D levels among US children aged 1 to 11 years: do children need more vitamin D? Pediatrics 2009; 124:1404-10.

22. Cashman KD, Dowling KG, Škrabáková Z, et al. Vitamin D deficiency in Europe: pandemic? Am J Clin Nutr 2016;103: 1033-44.

23. Maier GS, Horas K, Seeger JB, et al. Vitamin D insufficiency in the elderly orthopaedic patient: an epidemic phenomenon. Int Orthop 2015;39:787-92.

24. Weissmann R, Uziel Y. Pediatric complex regional pain syndrome: a review. Pediatr Rheumatol Online J 2016;14:29.

25. Joshi V, Hermann G, Balwani M, et al. Painless transient bone marrow edema syndrome in a pediatric patient. Skeletal Radiol 2014;43:1615-9.

26. Aigner N, Petje G, Schneider W, et al. Juvenile bone-marrow oedema of the acetabulum treated by iloprost. J Bone Joint Surg Br 2002;84:1050-2.

27. Pay NT, Singer WS, Bartal E. Hip pain in three children accompanied by transient abnormal findings on MR images. Radiology 1989;171:147-9.

28. Santori FS, Calvisi V, Manili M, et al. Regional migratory osteoporosis. Ital J Orthop Traumatol 1985;11:371-80.

29. Nicol RO, Williams PF, Hill DJ. Transient osteopaenia of the hip in children. J Pediatr Orthop 1984;4:590-2. 